\title{
Nanotechnology contributions to the pharmacotherapy of pediatric HIV: a dual scientific and ethical challenge and a still pending agenda
}

\section{"While a cure for HIV/AIDS remains a long-term commitment, our immediate and midterm challenges are to design cost-effective treatments and therapies with improved features."}

\section{KEYWORDS: cost-viable liquid formulations = efavirenz « ethical affordability * neglected diseases - pediatric HIV - scalable liquid formulations}

HIV/AIDS is the most deadly infectious disease of our time. It currently affects more than 40 million people worldwide [101]. HIV is a disease of poverty with more than $80 \%$ of the patients living in developing nations and $60 \%$ in the subSaharan region (sSR) of Africa. Children represent approximately $6 \%$ of the infected population, although $15 \%$ of new infections and $15 \%$ of the nearly 2 million annual deaths occur in children. In addition, 15 million children have been orphaned due to AIDS [102]; 11.6 million in the sSR. Pediatric HIV has been almost eradicated in developed countries by preventing the mother-tochild transmission and replacing natural delivery by cesarean. Conversely, in the developing world, 1000 new cases are registered every day. Only $10 \%$ of HIV-positive children have appropriate access to medication, this extent decreasing dramatically to less than $2 \%$ in the sSR [103]. Thus, while HIV is gradually becoming a chronic and manageable disease in Europe and North America owing to the high activity antiretroviral therapy or cocktail, most HIV-infected children in Africa will die before the age of 2 years [1].

\section{"Implementation of combined therapy is especially challenging in neonates and infants owing to the reduced number of antiretrovirals currently approved for pediatric administration."}

Implementation of combined therapy is especially challenging in neonates and infants owing to the reduced number of antiretrovirals (ARVs) currently approved for pediatric administration; only 12 of the 25 ARVs approved by the US FDA and EMA for adults have been clinically trialed and approved for children. Moreover, the commercial availability of liquid formulations is even more constrained, making dose adjustment and swallowing complicated. In cases where liquid formulations are not available, the only alternative to treat infants and young children is to develop extemporaneous formulations by manipulating the original solid forms $[2,3]$. These unlicensed medicines often display an unpredictable pharmacokinetic profile that may lead to subtherapeutic concentrations due to a lower oral bioavailability, or conversely, to severe adverse effects due to a greater absorption extent. In addition, their organoleptic properties are often neglected, regardless of the impact that patient compliance and adherence have on the therapeutic success. According to epidemiologists, adherence levels lower than 95\% (no more than two doses missed monthly in a twicea-day schedule) increase the chances of therapeutic failure to $50 \%[4,5]$. Clinicians agree that the strong bitterness of protease inhibitors (e.g., lopinavir) constitutes a key hurdle (if not the most remarkable) towards the fulfillment of the administration schedule of these first-choice ARVs in HIV-infected children. Moreover, the quality, safety and effectiveness of these medicines in countries with very limited infrastructures and fragile health systems are even more doubtful, this practice being strongly not recommended [6-8]. In this global context, the World Health Assembly (WHA) proclaimed the right of children to access safe, effective and proven medicines, and approved the resolution entitled 'Better medicines for children' in 2007 [104]. In addition, the global campaign 'Make medicines child size' has been launched [104]. The goal of this campaign is to provide an incentive to pharmaceutical companies and scientists in both industry and academia to develop medicines that fulfill the demands of pediatric patients in HIV, TB, HIV/TB coinfection and other neglected infectious diseases. A good example of the

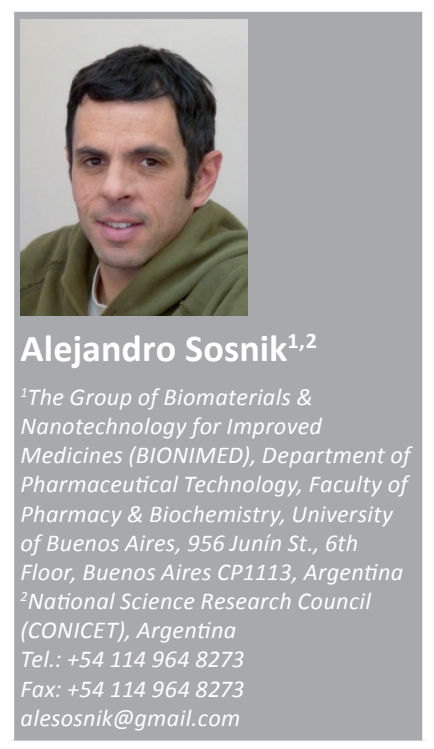


situation in pediatric HIV is the non-nucleoside reverse transcriptase inhibitor efavirenz. This very hydrophobic first-line ARV is recommended for the treatment of children above 3 years of age $[9,10]$. Owing to an extremely low aqueous solubility $(4 \mu \mathrm{g} / \mathrm{ml})$, a relatively limited oral bioavailability $(40-45 \%)$ is found [11]. In addition, the relatively high intersubject variability (54-58\%) shown by this ARV may result in serious adverse effects and, consequently, in treatment cessation [12]. To prevent this phenomenon, monitoring of plasma concentrations and dose fine tuning are strongly recommended [13-15]. However, this practice is not widespread and many children are potentially exposed to efavirenz overdose and unnecessary side effects that, in turn, might hinder the appropriate course of the treatment. The only liquid formulation commercially available (Sustiva ${ }^{\circledR}$ Oral Solution, Bristol-Myers) is a medium-chain triglyceride solution, which is not registered in all countries, for example Argentina [105]. This phenomenon constrains the access to only a limited number of patients that receive this medication directly from the producer under a compassionate status. Thus, a common practice in Argentina is to cut $600 \mathrm{mg}$ adult capsules into two halves and administer approximately $300 \mathrm{mg}$ a day. The efavirenz oral bioavailability with this formulation is $20 \%$ lower than that with capsules. Moreover, the intake of large volumes of medium-chain triglyceride produced reversible diarrhea and weight loss in rats [16]. Thus, this pharmaceutical excipient does not seem to be the most appropriate drug for children, especially in a chronic treatment. The reasons for this behavior are the extremely low aqueous solubility of the drug in water and the unmiscible nature of the vehicle employed.

\section{"Only 10\% of HIV-positive children have appropriate access to medication, this extent decreasing dramatically to less than $2 \%$ in the sub-Saharan region."}

Nanotechnology has contributed to improvements in the course of different diseases, such as cancer. Extensive research has been conducted over the years to develop better carriers that improve the biodistribution of antitumoral drugs and maximize their accumulation in diseased body sites, especially those resistant to the pharmacotherapy, while reducing the systemic exposure to these highly toxic compounds. These strategies also include the inhibition of efflux transporters, such as P-glycoprotein, that are involved in the development of resistance. Contrary to cancer, a disease affecting affluent and poor populations almost indistinctly, HIV shows a remarkably higher incidence in poor countries. Moreover, in children, the mortality toll is even higher than in the general population. Regretfully, innovation in infectious diseases of developing nations is not as profitable as in other diseases and pathologies. Thus, pharmaceutical companies do not invest significant efforts to improve their pharmacotherapy beyond the implementation of relatively simple (and not always effective) technological tools. In this framework, only a limited number of research groups worldwide are working towards solving the different limitations emerging in the pharmacotherapy of HIV in general and of pediatric HIV in particular.

\section{"...innovation is not always the best ally of nanotechnology for neglected diseases, as it usually implies expense and expense is tantamount to more constrained access."}

While a cure for HIV/AIDS remains a longterm commitment, our immediate and midterm challenges are to design cost-effective treatments and therapies with improved features. Based on the level of sophistication of the nanotechnological system, the main contributions of this emerging field to the pharmacotherapy of HIV could be classified into three main categories:

- Improvement of aqueous solubility, stability and consequently oral bioavailability. In this category, we could also envisage micro- and nano-encapsulation techniques to mask the unbearable taste of ARVs. Here, the involvement of nanotechnology appears more straightforward and feasible;

- Design of drug delivery systems to reduce the frequency of intake and, by doing so, enhance compliance and adherence;

- Targeting of viral reservoirs that are responsible for infection perpetuation and deleterious effects in CNS, where neural degeneration may develop and lead to death.

Realizing these challenges would transform a certainly deadly disease into a chronic one, regardless of the socioeconomic status of the patient. Owing to high costs, dosing obstacles, fewer approved drugs, limited number of liquid formulations and difficult registration issues, the situation for pediatric patients is more dramatic, and the gap between commercially 
available and appropriate formulations is enormous. Recently, the outlines of the immediate goals to improve the treatment of HIV-infected infants and children were stated [17]. However, it is worth mentioning that sophistication is usually accompanied by more difficult scale-up and higher production costs. In this context, I envisage that researchers in HIV and other neglected diseases face two major challenges. The first is a scientific challenge. However, we need to consider that since HIV mainly affects poor people, the expenses of the treatment will be covered by governments and nongovernmental organizations. Consequently, the second and equally crucial challenge is to make these new developments affordable to most of the patients. For example, with the aim of maintaining the solubility of efavirenz in water and improving its oral performance, our laboratory has employed a well-known Trojan horse technique, namely encapsulation within poly(ethylene oxide)poly(propylene oxide) block copolymer polymeric micelles [18,19]. One could ask: "Are these polymers novel?" The answer is short and simple: no. They are commercially available in a broad variety of molecular weights and compositions. One can buy them in large amounts as any pharmaceutical excipient. Since they have been shown to be biocompatible, some derivatives have been approved by the FDA as pharmaceuticals. I would say that these copolymers are nothing but the antithesis of any innovative material. We are currently working on the preparation of a protocol for a bioequivalence study in adult healthy volunteers. Therefore, why not try to tailor/design an innovative carrier that would fit the molecular features of the drug? We know how to do that and this would let us publish even more novel and elegant results. The answer is simple and probably provocative: any innovation in this regard would have a minor clinical relevance, as the chances of clinical evaluation and approval are, at least in Argentina, negligible. The same is valid for expensive biomaterials that would have a strong impact on the price of the medication. Most of the infected patients cannot afford that new formulation. I should confess that this is an internal debate that I face every single day. On one hand, I would want my research to be unique, different and more innovative. On the other, I would like to see my efforts 'crystallized' in a formulation that makes HIV-infected children's lives a little bit more comfortable and the treatment more compliant and consequently more successful. Very often, the tools (and the potential solutions) are right there on the shelf. They might not be elegant or innovative, but they are simple, scalable and more cost effective. In summary, innovation is not always the best ally of nanotechnology for neglected diseases, as it usually implies expense and expense is tantamount to more constrained access.

One could go one step further and analyze the affordability from an ethical perspective. My vision is that 'ethical affordability' in the context of ARV medicines has two dimensions.

\section{"Masking the taste of a protease inhibitor might seem a very trivial and even nonscientific challenge, but according to clinicians it is one of the main hurdles (if not the main) to be overcome in pediatric anti-HIV pharmacotherapy."}

The first dimension has to do with the affordability of new and more effective ARVs. New ARVs are remarkably more expensive than the older ones. For example, the price of treatment with zidovudine, the first ARV approved by the FDA in 1987, is today approximately US $\$ 100 /$ year/patient, while it used to be $\$ 8000$ in 1988. On the other side of the spectrum we find raltegravir, an integrase inhibitor approved by the FDA in 2007 that costs approximately $€ 10,000 /$ year/patient. The former is probably available to all patients, although it is less effective due to viral resistance. Conversely, the latter is only constrained to a reduced number of, more likely affluent, patients. As academia scientists, we probably have no relevant involvement in this matter. To find the equilibrium between profitability and appropriate access, prices need to be discussed between pharmaceutical companies and those facing the expenses of the treatment, namely governments and nongovernmental organizations.

The second dimension of affordability is related to the implementation of new technologies (e.g., nanotechnologies) to improve the performance of old ARVs. Thus, drawbacks such as limited oral bioavailability, unbearable taste, pill burden and viral resistance can be overcome by improving pharmacokinetic profiles, administration schedules and targeting viral sanctuaries. In this framework, we certainly are key players. However, we need to have in mind that this is not enough, as we are required to complement these developments with affordability. For instance, we should ask ourselves whether a hypothetical highly sophisticated zidovudine drug delivery system that enhances 
the performance of the drug substantially but, at the same time, dramatically increases its price is of clinical significance. Otherwise we are missing the chance of making a real contribution to the treatment of the disease.

In summary, we have witnessed tremendous progress in the medical implementation of nanotechnologies for the prevention, diagnosis and treatment of cancer. Conversely, in other specific niches such as HIV and TB, this progress has been not only at a much slower pace, but also remarkably more sporadic. Proof of this is given in the fact that, to the best of my knowledge, there are no nanotechnology products commercially available in the market for the treatment of HIV. A greater critical mass of scientists (and with them greater financial resources) will be undoubtedly required to achieve commensurable therapeutic breakthroughs on time. Moreover, a key feature is to understand and probably to internalize the need of the convergence of professionals with diverse backgrounds and from different societies that know, from first hand, the most immediate patient needs. In this regard, the generation of multicentered scientific networks that gather research groups with complementary expertise and know-how is a very positive move. This approach enables the optimized utilization of financial and, more importantly, human resources and will probably lead to a faster and a more vigorous and efficient response to patients' demands.

Masking the taste of a protease inhibitor might seem a very trivial and even nonscientific challenge, but according to clinicians it is one of the main hurdles (if not the main) to be overcome in pediatric anti-HIV pharmacotherapy. Can we afford to ignore these gross issues, while thinking of designing more sophisticated, innovative, unique and smart systems that are unfeasible owing to expensive costs? My opinion is that we should not. There are 2.5 million pediatric HIV patients ( $80 \%$ living in the sSR) and they deserve better medicines than we can surely develop. Thus, we should bear in mind that making these and other technological platforms affordable to patients is another supreme and undeniable challenge and, at the same time, a professional and ethical responsibility [20].

\section{"There are 2.5 million pediatric HIV patients (80\% living in the sSR) and they deserve better medicines than we can surely develop."}

Finally, a geriatric patient subpopulation is also emerging as a consequence of more successful combined pharmacotherapy; approximately 3 million patients over 50 years of age currently live with HIV. In this context, we will probably need to face and address drug interactions still unknown and to adjust the dose in cases of renal or liver disease. This will open new research avenues in the near future and enable further contributions of nanotechnologies. In any event, the implementation of creative (and not always very innovative) technological approaches should go towards the improvement of health and affordability for all infected patients.

\section{Financial \& competing interests disclosure}

The author has no relevant affliations or financial involvement with any organization or entity with a financial interest in or financial conflict with the subject matter or materials discussed in the manuscript. This includes employment, consultancies, honoraria, stock ownership or options, expert testimony, grants or patents received or pending, or royalties.

No writing assistance was utilized in the production of this manuscript.

\section{Bibliography}

1 HIVIAIDS in Europe: Moving From Death Sentence to Chronic Disease Management. Matic S, Lazarus JV, Donoghoe MC (Eds). World Health Organization Regional Office for Europe. World Health Organization Publications, Geneva, Switzerland (2006).

2 Standing JF, Tuleu C: Paediatric formulations - getting to the heart of the problem. Int. J. Pharm. 300, 56-66 (2005).

3 Committee on Pediatric AIDS (Section on international child health): Increasing antiretroviral drug access for children with HIV infection. Pediatrics 119, 838-845 (2007).
4 Panel of Clinical Practices for Treatment of HIV Infection: Guidelines for the use of antiretroviral agents to treat HIV infection in pediatric patients. Pan. Am. J. Public Health 10, 426-435 (2001).

5 Shah CA: Adherence to high activity antiretroviral therapy (HAART) in pediatric patients infected with HIV: issues and interventions. Indian J. Pediatr. 74, 55-60 (2007).

6 Choonara I, Conroy S: Unlicensed and off-label drug use in children: implications for safety. Drug Saf. 25, 1-5 (2002).

7 Nunn T, Williams J: Formulation of medicines for children. Br. J. Clin. Pharmacol. 59, 674-676 (2005).
8 Eileen Kairuz T, Gargiulo D, Bunt C et al: Quality, safety and efficacy in the 'off-label' use of medicines. Curr. Drug Safety 2, 89-95 (2007).

9 Barrueco N, Castillo I, Ais A et al.: Program of pharmaceutic attention to pediatric patients in antiretroviral therapy. Farm. Hosp. 29, 367-374 (2005).

10 Wintergerst U, Hoffmann F, Jansson A et al.: Antiviral efficacy, tolerability and pharmacokinetics of efavirenz in an unselected cohort of HIV-infected children. Antimicrob. Chemother. 61, 1336-1339 (2008). 
11 Gao JZ, Hussain MA, Motheram R et al:: Investigation of human pharmacoscintigraphic behavior of two tablets and a capsule formulation of a high dose, poorly water soluble/highly permeable drug (efavirenz). J. Pharm. Sci. 96, 2970-2977 (2007).

12 Fabbiani M, Di Giambenedetto S, Bracciale L et al: : Pharmacokinetic variability of antiretroviral drugs and correlation with virological outcome: 2 years of experience in routine clinical practice. J. Antimicrob. Chemother. 64, 109-117 (2009).

13 Csajka C, Marzollini C, Fattinger K et al:: Population pharmacokinetics and effects of efavirenz in patients with human immunodeficiency virus infection. Clin. Pharm. Ther. 73, 20-30 (2003).

14 Aarnoutse R, Schapiro JM, Bouchr CAB et al: : Therapeutic drug monitoring. Drugs 63, 741-753 (2003).

15 van Luin M, Gras L, Richter C et al. Efavirenz dose reduction is safe in patients with high plasma concentrations and may prevent efavirenz discontinuations. J. Acquir. Immune Defic. Syndr. 52, 240-245 (2009).
16 Sellers RS, Antman M, Phillips J et al.: Effects of Miglyol 812 on rats after 4 weeks of gavage as compared with methylcellulose/ Tween 80. Drug. Chem. Toxicol. 28, 423-432 (2005).

17 Dionisio D, Gass R, McDermott P et al.: What strategies to boost production of affordable fixed-dose anti-retroviral drug combinations for children in the developing world? Current HIV Res. 5, 155-187 (2007).

18 Chiappetta DA, Hocht C, Taira C, Sosnik A: Efavirenz-loaded polymeric micelles for pediatric anti-HIV pharmacotherapy with significantly higher oral bioavailability. Nanomedicine (Lond.) 5 , 11-23 (2010).

19 Chiappetta DA, Hocht C, Sosnik A: A highly concentrated and taste-improved aqueous formulation of efavirenz for a more appropriate paediatric management of the anti-HIV therapy. Curr. HIV Res. 8, 23-31 (2010).

20 Sosnik A, Amiji M: Nanotechnology solutions for infectious diseases in developing nations. Adv. Drug Del. Rev. 62, 375-377 (2010).

\section{Websites}

101 AIDS epidemic update 2007, World Health Organization www.who.int/hiv/epiupdates/en/index.html (Accessed May 2010)

102 AIDS orphans, AVERT Organization www.avert.org/aidsorphans.htm (Accessed May 2010)

103 The US President's Emergency Plan for AIDS Relief (PEPFAR). Pediatric treatment and care www.pepfar.gov/press/86524.html (Accessed May 2010)

104 WHO/Make medicines child size www.who.int/childmedicines/en (Accessed May 2010)

105 Administración Nacional de Medicamentos, Alimentos y Tecnología Médica (ANMAT), Ministry of Health, Argentina www.anmat.gov.ar (Accessed May 2010) 